\title{
Raça e desigualdade entre as mulheres: um exemplo no sul do Brasil
}

\author{
Race and inequality among women: \\ an example in southern Brazil
}

Maria Teresa Anselmo Olinto 1,2

Beatriz Anselmo Olinto 3

\footnotetext{
1 Centro de Ciências da Saúde, Universidade do Vale do Rio dos Sinos. Av. Unisinos 950, C.P. 275 , São Leopoldo, RS 93022-000, Brasil. mtolinto@cirrus.unisinos.br 2 Programa de Pós-Graduação em Epidemiologia Universidade Federal de Pelotas. C.P. 464 , Pelotas, RS 96001-970, Brasil. 3 Departamento de Ciências Humanas, Universidade Estadual do Centro-oeste. C.P. 730, Guarapuava, PR 85015-430, Brasil. cch@unicentro.br
}

\begin{abstract}
This study uses epidemiological data to investigate socioeconomic proportions of distinctions raised by "racism" in Brazilian society. A population-based cross-sectional study was conducted with a sample of 2,779 women ages 14 through 49, living in a southern Brazilian city. Black and mixed-race women had less schooling, lower family income, and worse housing conditions than white women. They also used contraceptive methods less frequently, had more children, and had higher spontaneous abortion and stillbirth rates than white women. Virtually all of the results show a linear relationship between such categories, i.e., the "darker" the woman's skin color, the worse her socioeconomic and reproductive conditions. We also observed that black women were either separated, divorced, or widowed, another apparent factor for black women's impoverishment, related mainly to their limited employment opportunities. The results of the current study indicate that racial relations among women are an issue that should foster a discussion concerning citizenship in Brazil.
\end{abstract}

Key words Race; Ethnic Groups; Women's Health

Resumo Através de dados epidemiológicos este estudo pretende evidenciar as proporções sócioeconômicas das distinções criadas pelo "racismo contemporizador" da sociedade brasileira. Foi realizado um estudo transversal de base populacional com uma amostra representativa de 2.779 mulheres, de 15 a 49 anos vivendo em uma cidade no sul do Brasil. As mulheres negras e pardas apresentaram menor escolaridade, renda familiar, piores condições de moradia do que as mulheres brancas. Ao mesmo tempo, usavam menos métodos contraceptivos, tinham mais filhos $e$ apresentavam maior perda fetal do que as mulheres brancas. Chama a atenção que praticamente todos esses resultados apresentaram tendência linear entre as categorias, isto é, à medida que havia um "escurecimento" da pele, piores ficavam as condições sócio-econômicas das mulheres. Também foi observado que as mulheres negras eram mais separadas, divorciadas ou viúvas, evidenciando mais um aspecto de pauperização das mulheres negras, principalmente pelo limitado acesso dessas ao mercado de trabalho. Os resultados deste estudo demostram que as relações raciais entre as mulheres são uma problemática que deve permear a discussão sobre cidadania no Brasil.

Palavras-chave Raça; Grupos Étnicos; Saúde da Mulher 


\section{Introdução}

As desigualdades sociais entre as regiões do Brasil têm sido amplamente descritas. Diversos trabalhos apontam para melhores indicadores nas regiões Sul e Sudeste comparado com o restante do país. O Sul, por exemplo, apresenta a maior esperança de vida ao nascer, menor mortalidade infantil e uma das menores taxas de analfabetismo do país. Esses dados têm sido analisados, na maioria das vezes, como expressão de uma suposta melhor qualidade de vida na Região Sul.

Mas, diferenciais também estão presentes quando observa-se categorias dentro de uma mesma região. No Sul embora a taxa de analfabetismo geral seja relativamente baixa, é cerca de $30 \%$ maior para as mulheres do que para os homens (BEMFAM, 1997). Em relação à renda, em 1991, o rendimento médio dos chefes de domicílios, homens moradores da zona urbana, foi cerca de duas vezes maior do que das mulheres, respectivamente, 4,2 e 2,4 salários mínimos. Assim, mesmo que em média as mulheres do Rio Grande do Sul apresentem melhores indicadores do que aquelas vivendo em outros estados do país, encontram-se em situação inferiorizada se comparadas com os homens da mesma região.

Além das desigualdades entre regiões ou gêneros devem ser articuladas outras categorias de análise que demonstrem diferenças dentro destas diferenças, ou seja, aquelas que existem dentro de um mesmo gênero: como classe, raça, etnia, espaço/região, etc (Scott, 1992). Este estudo, restringindo-se a uma amostra de mulheres residentes na cidade de Pelotas, Rio Grande do Sul, e controlando para fatores regionais e diferenças entre os gêneros, pretende evidenciar as distâncias sócio-econômicas e demográficas construídas através da percepção de "raças".

\section{Metodologia}

A partir de uma amostra de 2.779 mulheres de 15 a 49 anos, residentes na zona urbana da cidade de Pelotas, foram estudados os diferenciais sócio-econômicos, demográficos e reprodutivos segundo a "raça".

O delineamento utilizado foi de um estudo transversal de base populacional. O cálculo do tamanho de amostra foi obtido para atingir dois objetivos, sendo um deles a descrição das características sócio-econômicas e demográficas de uma amostra representativa de mulheres em idade reprodutiva na referida cidade. Em outra publicação da autora foram apresenta- dos o cálculo e o processo amostral detalhadamente (Olinto \& Galvão, 1999).

O tamanho total da amostra inicial foi de 3.002 mulheres - excluindo $5 \%$ entre recusas e perdas. Dessas, devido a uma falha na impressão dos questionários, para 223 mulheres não foram coletadas as informações sobre a raça. Esse erro não foi sistemático, conseqüentemente não afetando a distribuição desta variável. Cálculos posteriores mostraram que o número de mulheres estudadas ( $\mathrm{n}=2.779$ ) foi suficiente para atingir os objetivos do estudo.

A equipe de trabalho de campo incluiu 14 entrevistadoras, sendo todas do sexo feminino e cursando alguma faculdade na área de saúde. Foi utilizado como instrumento de pesquisa um questionário estruturado, pré-codificado contendo 95\% das questões fechadas, que abordavam características sócio-econômicas, demográficas e reprodutivas das mulheres.

A variável raça foi avaliada conforme referência das entrevistadoras classificando-se em: branca, "parda” e negra. Essa classificação foi construída a partir da percepção de características fenótipas, como o tom de pele e características do cabelo, de forma racializada. Cabe salientar que durante as revisitas do controle de qualidade não foi observado nenhum desacordo com a classificação racial estabelecida na primeira entrevista.

Quanto às raças consideradas "amarela” ou indígena, no estado do Rio Grande do Sul o porcentual apresentado pela Fundação Instituto Brasileiro de Geografia e Estatística (IBGE) é de apenas 0,5\% (IBGE, 1991). Por ser um número pequeno, no presente estudo essas foram incluídas como "pardas". Análises estatísticas paralelas mostraram que a exclusão destas mulheres da categoria "pardas" não teriam alterado as tendências apontadas nos resultados.

O controle de qualidade dos dados foi garantido através de cinco mecanismos, que incluíram desde a revisão precoce dos questionários, supervisão de codificação, 5\% de revisitas, criação de banco de dados com checagem da consistência das informações e dupla entrada de dados. A análise dos dados restringiu-se a descrição das características das mulheres segundo as três categorias propostas de "raça", através de freqüências simples e comparação entre médias ou proporções.

\section{Resultados}

Foram estudadas 2.779 mulheres, sendo destas $2.350(84,6 \%)$ brancas, 215 (7,7\%) "pardas" e 214 $(7,7 \%)$ negras. A Tabela 1 apresenta algumas 
Médias e percentuais das principais características sócio-econômicas e demográficas segundo a raça das 2.779 mulheres de 15 a 49 anos $^{1}$.

\begin{tabular}{|c|c|c|c|c|}
\hline Características & Brancas & Mulatas & Negras & p-valor \\
\hline Idade média (em anos) & $30,9(2.350)$ & $31,2(215)$ & $31,3(214)$ & 0,70 \\
\hline \multirow[t]{2}{*}{ Escolaridade média (anos completados) } & $8,8(2.350)$ & $6,9(215)$ & $6,6(214)$ & $<0,001$ \\
\hline & & & & $<0,0012$ \\
\hline \multirow[t]{2}{*}{ Renda familiar (em salários mínimos) } & $10,4(2.331)$ & $6,7(212)$ & $4,1(212)$ & $<0,001$ \\
\hline & & & & $<0,0012$ \\
\hline Estado civil & $(2.350)$ & (215) & (214) & \\
\hline Casada & $48,7 \%$ & $43,7 \%$ & $29,4 \%$ & $<0,001$ \\
\hline Em união & $82, \%$ & $11,2 \%$ & $13,6 \%$ & $<0,0012$ \\
\hline Solteira & $34,5 \%$ & $37,2 \%$ & 43,55 & \\
\hline Viúva & $1,7 \%$ & $2,3 \%$ & $4,7 \%$ & \\
\hline Separada/divorciada & $6,9 \%$ & $5,6 \%$ & $8,9 \%$ & \\
\hline Atividade & $(2.350)$ & (215) & (214) & \\
\hline Estuda & $18,4 \%$ & $10,7 \%$ & $10,7 \%$ & $<0,001$ \\
\hline Trabalha & $4,3 \%$ & $46,0 \%$ & $55,0 \%$ & 0,492 \\
\hline Estuda e trabalha & $5,7 \%$ & $11,2 \%$ & $7,5 \%$ & \\
\hline Dona de casa & $32,3 \%$ & $31,6 \%$ & $25,7 \%$ & \\
\hline \multirow[t]{2}{*}{ Água encanada dentro de casa } & $96,9 \%(2.341)$ & $92,0 \%(213)$ & $82,0 \%(209)$ & $<0,001$ \\
\hline & & & & $<0,0012$ \\
\hline \multirow[t]{2}{*}{ Sanitário com descarga } & $95,7 \%(2.341)$ & $85,9 \%(213)$ & $80,4 \%(209)$ & $<0,001$ \\
\hline & & & & $<0,0012$ \\
\hline
\end{tabular}

1 Os números entre parênteses representam os números absolutos de mulheres que foram incluídas em cada categoria;

2 Teste para tendência linear.

características sócio-econômicas e demográficas segundo a raça. Como seria esperado devido ao processo amostral, não houve diferença significativa entre a idade média de cada grupo de raça. Em relação à escolaridade e à renda familiar constatou-se diferenças estatisticamente significativas e com tendência linear. As mulheres brancas apresentaram em média 8,8 anos de escolaridade, valor superior tanto para as mulheres "pardas" $(6,9)$, como para as negras $(6,6)$. A renda familiar evidenciou mais esses diferenciais, as mulheres brancas apresentavam renda familiar cerca de 1,5 vezes maior do que as pardas e 2,5 maior do que as negras. Ainda é possível observar que, embora em Pelotas 95\% dos domicílios disponham de água dentro de casa, para as mulheres negras esse índice caiu para $82 \%$. O porcentual de domicílios apresentando sanitário com descarga, foi de 95,7\%, 85,9\% e $80,4 \%$, respectivamente, para aqueles nos quais residiam mulheres brancas, "pardas" e negras.
Quanto ao estado civil, podemos observar que as mulheres "pardas" e principalmente as negras apresentaram mais uniões "não formais" do que as mulheres brancas (Tabela 1). Também chama atenção o maior porcentual de mulheres viúvas tanto para as mulheres pardas como, principalmente, para as negras. A mesma tendência ocorre em relação ao número de mulheres separadas e divorciadas. Assim, observa-se as mulheres negras vivendo mais sozinhas do que as pardas e brancas, $13,6 \%, 7,9 \%$ e $8,6 \%$, respectivamente.

Os diferenciais por raça persistem quando observa-se as características reprodutivas. A fim de possibilitar a comparação com outros estudos, a Tabela 2 apresenta todas as variáveis com dois tipos de denominador: (a) considerando todas as mulheres e (b) apenas as mulheres que haviam iniciado sua vida sexual. $\mathrm{O}$ menor número de filhos é observado nas mulheres brancas, justamente aquelas com maior 
Médias e percentuais das principais características reprodutivas segundo a raça das 2.779 mulheres de 15 a 49 anos¹.

\begin{tabular}{|c|c|c|c|c|}
\hline Características & Brancas & Mulatas & Negras & $p$-valor \\
\hline \multirow[t]{2}{*}{ Média de filhos (a) } & $1,3(2.341)$ & $1,4(212)$ & $1,7(214)$ & $<0,001$ \\
\hline & & & & $<0,0012$ \\
\hline \multirow[t]{2}{*}{ Média de filhos (b) } & $1,5(2.026)$ & $1,6(181)$ & $2,1(179)$ & $<0,001$ \\
\hline & & & & $<0,0012$ \\
\hline \multirow[t]{2}{*}{ Média de gestações (a) } & $1,6(2.350)$ & $1,9(212)$ & $2,2(214)$ & $<0,001$ \\
\hline & & & & $<0,0012$ \\
\hline \multirow[t]{2}{*}{ Média de gestações (b) } & $1,9(2.035)$ & $2,1(184)$ & $2,6(179)$ & $<0,001$ \\
\hline & & & & $<0,0012$ \\
\hline $\begin{array}{l}\text { Mulheres não utilizando } \\
\text { métodos anticoncepcionais (a) }\end{array}$ & $28,4 \%(2.035)$ & $31,8 \%(211)$ & $39,8 \%(211)$ & $\begin{array}{l}0,07 \\
0,042\end{array}$ \\
\hline Mulheres não utilizando & $19,5 \%(1.995)$ & $22,5 \%(182)$ & $29,7 \%(175)$ & 0,06 \\
\hline métodos anticoncepcionais (b) ${ }^{3}$ & & & & 0,042 \\
\hline \multirow[t]{2}{*}{ Mulheres esterilizadas (a) } & $10,3 \%(2.350)$ & $10,7 \%(215)$ & $12,1 \%(214)$ & 0,68 \\
\hline & & & & 0,402 \\
\hline \multirow[t]{2}{*}{ Mulheres esterilizadas (b) } & $11,8 \%(2 ; 305)$ & $12,5 \%(184)$ & $14,5 \%(179)$ & 0,56 \\
\hline & & & & 0,302 \\
\hline \multirow[t]{2}{*}{ Perdas fetais (a) } & $20,9 \%(2.350)$ & $26,5 \%(215)$ & $27,6 \%(214)$ & 0,02 \\
\hline & & & & 0,022 \\
\hline \multirow[t]{2}{*}{ Perdas fetais (b) } & $24,2 \%(2.035)$ & $31,8 \%(184)$ & $33,0 \%(179)$ & $<0,01$ \\
\hline & & & & $<0,012$ \\
\hline
\end{tabular}

1 Os números entre parênteses representam os números absolutos de mulheres

que foram incluídas em cada categoria;

2 Teste para tendência linear;

3 Foram excluídas as mulheres que estavam na menopausa;

(a) considerando todas as mulheres e (b) apenas as mulheres que haviam iniciado sua vida sexual.

escolaridade, melhor renda e melhores condições vida. Entre todas as mulheres negras, cerca de $40 \%$ não usavam nenhum método contraceptivo. Não houve diferença do porcentual de mulheres esterilizadas entre as raças. Considerando apenas as mulheres negras que tinham vida sexual ativa, um terço delas relataram perda fetal, resultado muito similar ao apresentado pelas mulheres "pardas" $(31,8 \%)$, e superior aquele referente às mulheres brancas $(24,2 \%)$.

\section{Discussão}

A opção pela análise de desigualdades e também pelo viés das relações raciais vem lançar um olhar sobre uma questão respondida por muito tempo pelos mitos de "democracia ra- cial" - o silêncio imposto sobre o preconceito racial no país (Munanga, 1996). Não pretendeu-se aqui analisar o referido mito, nem como um instrumento ideológico e nem buscar as suas re-significações cotidianas, mas sim, demonstrar através de dados epidemiológicos, quais as proporções sócio-econômicas das distinções criadas pelo racismo contemporizador da sociedade brasileira (Pereira, 1996).

Tem-se consciência que os resultados deste estudo são apenas uma pequena parte do abismo social e econômico existente entre "raças" no Brasil. Embora com as limitações da variável "raça" utilizada neste estudo foi possível mostrar alguns diferenciais importantes que permeiam a sociedade pesquisada.

Como um país que recebeu imigrantes das mais variadas regiões do mundo, a população 
brasileira vem sendo identificada como miscigenada. Dentro destas condições, os processos de identificação das diferenças entre grupos humanos, passam pelas diferenças fenótipas e culturais, sempre de maneira relacional em construções dinâmicas de "nós" e "outros" (Poutignat, 1998). Assim a composição da categorização das entrevistadas em "brancas”, "pardas" e "negras" deve ser considerada a partir do contexto sócio-cultural das entrevistadoras e não como categorias fechadas.

Esta sensibilidade contemporânea a certas nuances fenótipas são social e historicamente construídas - a partir da expansão colonial européia - incidindo nas identificações de grupos humanos e balizando relações sociais. Destaca-se aqui, as concepções de "cor" e/ou "raça" perspassando o acesso a melhores condições sociais e econômicas, bem como ao mercado de trabalho. Desta forma, se a viabilidade da categoria raça em sua vertente biológica no que tange os seres humanos é questionável, a análise das relações raciais nas sociedades permanece uma problemática pertinente (Santos, 1996).

O presente estudo encontrou um porcentual de mulheres negras superior ao fornecido pelo IBGE (1997) para o Rio Grande do Sul $(7,7 \%$ versus $3,1 \%$ ) e menor porcentual de mulheres pardas $(7,7 \%$ versus $11 \%)$. Essas diferenças podem ser consideradas como conservadoras para as comparações apresentadas aqui. No presente estudo algumas mulheres pardas podem ter sido percebidas pelas entrevistadoras como negras. Isto leva a pensar que as condições demográficas, sócio-econômicas e reprodutivas das mulheres negras podem ainda ser piores do que as encontradas. Por outro lado, se a identificação de "raça" ou de "cor" fossem feitas pelas próprias entrevistadas a tendência seria de uma maior diversidade de classificação e nuan- ces (Maggie, 1996; Sansone, 1996; Williams, 1997).

Outro fator levantado foi o elevado número de mulheres negras vivendo sozinhas, considerando-se as viúvas, separadas e divorciadas. Observa-se nestes dados a ampliação do espaço da mulher como chefe de domicílio, que frente as limitadas possibilidades de acesso daquelas ao mercado de trabalho, permite que seja vislumbrado mais um aspecto do processo de pauperização e exclusão social deste grupo.

Contribuindo para esta análise, foi detectado o menor uso de métodos contraceptivos por mulheres negras e pardas do que brancas. Esse fator expressa muito mais a iniqüidade ao acesso de métodos e serviços existentes do que uma escolha pessoal dessas mulheres. Embora alguns estudos realizados no Brasil mostrem um maior número de ligaduras tubárias entre mulheres negras, esse resultado não foi encontrado aqui. Estes dados são compatíveis com outro estudo realizado no Rio Grande do Sul (Diasda-Costa \& Olinto, 1999).

Os resultados desta pesquisa enfatizam que, para uma apreensão da complexidade das experiências sociais femininas não basta perceber as diferenciações de gênero, é necessário ir além e, interpelar outros aspectos do viver social, entre eles os de identificação racial. Os resultados desiguais entre brancas, "pardas" e negras obtidos por esta pesquisa demonstram que as relações raciais são uma problemática que deve permear a discussão sobre cidadania no Brasil. A compreensão da exclusão social, não pode ser limitada por generalizações que trabalhem apenas com categorias regionais ou de gênero que silenciam as diferenças na diferença. Somente um olhar que perceba as imbricadas clivagens que permeiam estes espaços pode construir respostas pertinentes.

\section{Referências}

BEMFAM (Sociedade Civil Bem-Estar Familiar no Brasil), 1997. Pesquisa Nacional sobre Demografia e Saúde - 1996. Rio de Janeiro: BEMFAM.

DIAS-DA-COSTA, J. S. \& OLINTO, M. T. A., 1999. Ligadura tubária: Uma representação da iniqüidade do acesso ao sistema de saúde em Pelotas, RS. Revista de Ginecologia \& Obstetrícia, 10:130-134.

HASENBALG, C., 1996. Entre o mito e os fatos: Racismo e relações raciais no Brasil. In: Raça, Ciência e Sociedade (M. C. Maio \& R. V. Santos, org.), pp. 235-249, Rio de Janeiro: Fundação Oswaldo Cruz/ Centro Cultural Banco do Brasil. 
IBGE (Fundação Instituto Brasileiro de Geografia e Estatística), 1991. Censo Demográfico 1991. Rio de Janeiro: IBGE.

IBGE (Fundação Instituto Brasileiro de Geografia e Estatística), 1997. Pesquisa Nacional por Amostra de Domicílios 1996. CD-ROM. Rio de Janeiro: IBGE.

MAGGIE, Y., 1996. Aqueles a quem foi negada a cor do dia: As categorias cor e raça na cultura brasileira. In: Raça, Ciência e Sociedade (M. C. Maio \& R. V. Santos, org.), pp. 225-234, Rio de Janeiro: Fundação Oswaldo Cruz/Centro Cultural Banco do Brasil.

MUNANGA, K., 1996. As facetas de um racismo silenciado. In: Raça e Diversidade (L. M. Schwarcz \& R. S. Queiroz, org.), pp. 213-229, São Paulo: Edusp.

OLINTO, M. T. A. \& GALVÃO, L. W., 1999. Características reprodutivas de mulheres de 15 a 49 anos: Estudos comparativos e planejamento de ações. $R e$ vista de Saúde Publica, 33:64-72.

PEREIRA, J. B. B., 1996. O retorno do racismo. In: Raça e Diversidade (L. M. Schwarcz \& R. S. Queiroz, org.), pp. 17-27, São Paulo: Edusp.
POUTIGNAT, P. \& STREIFF-FENART, J., 1998. Teorias da Etnicidade. São Paulo: Universidade Estadual Paulista.

SANSONE, L.,1996. As relações raciais em casa-grande e senzala revisitadas à luz do processo de internacionalização e globalização. In: Raça, Ciência e Sociedade (M. C. Maio \& R. V. Santos, org.), pp. 207-217, Rio de Janeiro: Fundação Oswaldo Cruz/Centro Cultural Banco do Brasil.

SANTOS, J. R., 1996. O negro como lugar. In: Raça, Ciência e Sociedade (M. C. Maio \& R. V. Santos, org.), pp. 219-223, Rio de Janeiro: Fundação Oswaldo Cruz/Centro Cultural Banco do Brasil.

SCOTT, J., 1992. História das Mulheres. In: A Escrita da História (P. Burke, ed.), pp. 63-95, São Paulo: Universidade Estadual Paulista.

WILLIANS, D., 1997. Race and health: Basic questions, emerging directions. Annals of Epidemiology, 7: 322-333. 